\title{
Consequences of display changes during interrupted visual search: Rapid resumption is target specific
}

\author{
Alejandro Lleras \\ University of Illinois at Urbana-Champaign, Urbana, Illinois \\ AND \\ RonAld A. RENSINK AND JAMES T. ENNS \\ University of British Columbia, Vancouver, British Columbia
}

\begin{abstract}
Visual search can be resumed more rapidly following a brief interruption to an old display than it can be initiated on a new display, pointing to a critical role for memory in search (Lleras, Rensink, \& Enns, 2005). Here, we examine how this rapid resumption is affected by changes made to the display during the interruption of search. Rapid resumption was found to depend on the prior presentation of the target, not merely the distractor items (Experiment 1), and was unaffected by the relocation of all distractor items (Experiment 2). Further, whereas changes to response-irrelevant features of the target did not eliminate rapid resumption (Experiment 3), changes to response-relevant features did (Experiment 4). These results point to the target specificity of rapid resumption and are consistent with reentrant theories of visual awareness.
\end{abstract}

Recent findings have begun to clarify the role that various memory systems play in visual search. For example, there is now significant evidence that spatial working memory is recruited during visual search (e.g., Oh \& Kim, 2004; Woodman \& Luck, 2004) and it is most likely involved in keeping track of spatial locations that have been visited by attention (e.g., McCarley, Wang, Kramer, Irwin, \& Peterson, 2003; Peterson, Kramer, Wang, Irwin, \& McCarley, 2001; Takeda \& Yagi, 2000). It also appears to be involved in the planning of which locations to attend next, as though search was based on a "foraging facilitator" mechanism (Klein \& McInnes, 1999). Meanwhile, executive working memory is also recruited during visual search tasks (e.g., Han \& Kim, 2004), most likely as a means for keeping task instructions active in working memory and for maintaining a visual image or "template" of the target that can be used to influence the deployment of attention in the scene (e.g., Downing, 2000; Fockert, Rees, Frith, \& Lavie, 2001). But it is also important to note that not everything is remembered in search. For example, participants do not make use of information about rejected distractors (Horowitz \& Wolfe, 1998, 2001, 2003 ), pointing to an important limit in the nature of what is remembered during search.

In a recent study, Lleras, Rensink, and Enns (2005) used an interrupted search paradigm to demonstrate yet another contribution of memory. In that task, participants were presented with only brief glimpses of the search display (typical "look" times were $100 \mathrm{msec}$ ), with these glimpses alternating with blank screens of longer durations (typical "wait" times were $900 \mathrm{msec}$ ). Despite the difficulty of this task, participants were generally very good at completing it: participants correctly identified the target after only three presentations of the display on more than $75 \%$ of the trials and with accuracy levels above $95 \%$. The most surprising aspect of their performance, in light of current theories, was that participants often showed extremely short response latencies to target identification following re-presentation of the display (below $400 \mathrm{msec}$ ), a finding Lleras et al. referred to as rapid resumption.

To be certain, not all responses following interruptions were fast. A more detailed analysis of the response time (RT) distributions showed that, following interruptions, there appeared be two distinct phases: an early phase peaking in the range of $200-300 \mathrm{msec}$, and a later phase peaking in the range of $600-800 \mathrm{msec}$. As an illustration, Figure 1 (based on data from Lleras et al., 2005) shows the RT distributions following the first presentation of an interrupted search display (panel A), and following all subsequent re-presentations of the search display (panel B). Lleras et al. interpreted the early phase (rapid resumption) as evidence that some perceptual preprocessing ${ }^{1}$ of the search display had occurred during the preceding look. As a result, fast RTs were observed whenever that preprocessing included information about the target, whereas slower RTs were observed otherwise. In other words, partial in-

A. Lleras, alleras@uiuc.edu 

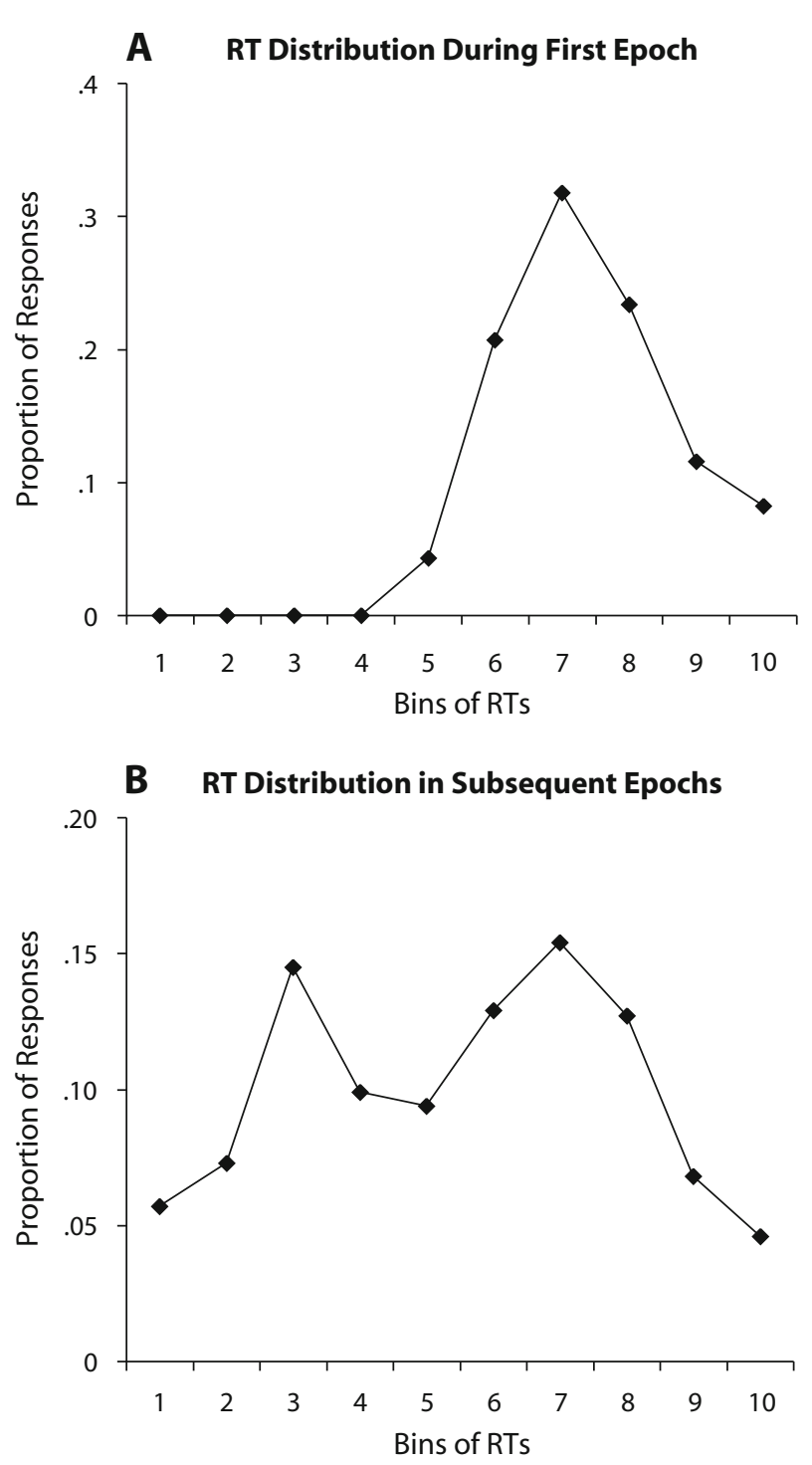

Figure 1. Correct RT distributions in an interrupted search task, adapted from Lleras, Rensink, and Enns (2005). Panel A presents the distribution of RTs following the first presentation of the search display. Panel B presents the combined distribution of RTs following all subsequent presentations of the search display.

formation about some subset of items in the display was kept in memory during the interruption even though participants were totally unaware of this (see Experiment 6 of Lleras et al., 2005).

The goal of the present paper was to investigate both the nature and the content of the target preprocessing that leads to rapid resumption. The original interpretation given by Lleras et al. (2005) was inspired by recent theoretical developments in the field of visual backward masking (Di Lollo, Enns, \& Rensink, 2000; Enns \& Di Lollo, 2000). In brief, they proposed that the preprocessing involves the formation of a perceptual hypothesis regarding the location and attributes of the target (e.g., color, shape) and that this hypothesis interacts with sensory processes upon re-presentation of the search display. The experiments here examined this proposal in further detail.

\section{OVERVIEW OF THE PRESENT STUDY}

Experiments 1 and 2 tested whether rapid resumption is a behavioral index of target-specific preprocessing. In particular, they tested the claim that the preprocessing involves information specific to the target object, rather than, for example, more general information about the configuration of the display items, or the instructions in the task. In Experiment 1, we gave observers the opportunity to preprocess everything about the search display, except for the target. The results showed that rapid resumption was absent in those conditions. In Experiment 2, we changed the locations of the distractors between looks while keeping target location and identity constant. The results showed that rapid resumption still occurred under these conditions. Both results support the idea that the preprocessing involves target-specific information.

Experiments 3 and 4 investigated the contents of the preprocessing by changing specific features of the target item between looks at the search array. Our reasoning was that if rapid resumption depended on a specific piece of preprocessed information, then changing that information between looks would likely reduce, delay or even eliminate rapid resumption. To anticipate, rapid resumption was still observed when features of the target not critical to the response (i.e., task irrelevant) were changed between looks. Meanwhile, rapid resumption was eliminated when the features of the target that changed were critical (i.e., were task relevant).

\section{EXPERIMENT 1}

To test whether rapid resumption is due to targetspecific preprocessing, we intermixed trials in which the target was always present ("target in first look") with trials in which the target was absent in the first display, but present in all subsequent re-presentations ("target in second look"). If rapid resumption were due to preprocessing that is not specific to the target, it ought to be observed to the same extent in both conditions. But if it is specific to the target, it ought to be absent after the second display presentation in the "target-in-second-look" condition because at that point, participants have not yet had a chance to preprocess any target information.

It should be noted that the methodology of rapid resumption bears some surface similarity to that of another visual phenomenon, known as visual marking (Watson \& Humphreys, 1997). Visual marking refers to the finding that previewing a subset of the distractors in a difficult search task significantly increases the efficiency of target identification (Watson \& Humphreys, 1997). This seems to come about both because the previewed distractors are discounted or "marked" in some way (Kunar, Humphreys, \& Smith, 2003) and because the later appearing items are privileged by virtue of the luminance transients associated with their onset (Donk \& Verburg, 2004). In the present 
experiment, the "target-in-second-look" condition essentially permits observers to preview all items other than the target. If rapid resumption is simply another benefit of this preview, a presentation of the distractors alone should result in a significant early phase of RT responses. But if rapid resumption involves target preprocessing then the "target-in-second-look" condition should not lead to early-phase RTs.

\section{Method}

Participants. Fifteen undergraduate students from the University of Illinois participated in this experiment in exchange for extra credit in an introduction to psychology course. All participants reported normal or corrected-to-normal visual acuity.

Apparatus. Stimuli were presented on a 17-in. color monitor controlled by a Pentium-based computer. Responses were gathered with a standard keyboard. Displays consisted of $15^{~ " L " ~ i t e m s ~}\left(0.8^{\circ} \times\right.$ $0.8^{\circ}$ of visual angle, evaluated at fixation, from a viewing distance of $57 \mathrm{~cm}$ ) that were presented randomly within a $6 \times 6$ grid of possible locations, and one " $T$ " item of identical size. The Ls and the $\mathrm{T}$ could be oriented in one of four possible orientations (upright, upside down, pointing right or pointing left) and were either red or blue, such that they were always an identical number of red and blue items in a search display. The total search grid occupied 14.7 degrees of visual angle.

Task and Design. The participant's task was to report the color of the $\mathrm{T}$ item as rapidly and accurately as possible. Participants used the keyboard to report target color: the "z" key to indicate blue targets and the "/" key to indicate red targets. Participants were encouraged to respond as quickly as possible, while maintaining a level of accuracy above $95 \%$. The experiment consisted of a one-factor, withinsubjects design. The independent variable was target look, which had two levels (target in first look and target in second look). Both conditions occurred equally often and were equally likely to occur within a block of trials. Participants completed 10 blocks of 64 trials each for a total of 320 trials per subject per condition.

Procedure. Each trial started with the presentation of a small fixation cross at the center of the screen. After $500 \mathrm{msec}$, the fixation cross disappeared and was replaced by the first search display. This was presented for $100 \mathrm{msec}$, which we refer to as "look" time. The search display was then replaced by a blank screen for a duration of $900 \mathrm{msec}$, which we refer to as "wait" time. We refer to the two display sequence of one search and one blank display as an "epoch" (total duration, 1,000 msec). This sequence was repeated 16 times or until a response was recorded, whichever happened first. In the target-in-second-look condition, the target $\mathrm{T}$ was not presented in the first presentation of the search display, but appeared on every presentation of the display afterward, at a previously unoccupied location. Otherwise, the display remained unchanged in each look.

\section{Results}

Table 1 contains the frequency of correct responses in each target look condition as a function of $100-\mathrm{msec}$ bins following the onset of the first display. These raw data were summarized by compiling normalized RT distributions for the first, second, and third epochs in each of the two conditions (the observed frequency in each bin divided by the total number of trials in the condition). The resulting distributions are shown in Figure 2. Panel A shows the RT distributions obtained during the first epoch (i.e., following the first look at the display). The distribution for target-in-first-look condition $(n=775)$ was typical of that observed in the interrupted search paradigm, (see Lleras et al., 2005): almost no responses were recorded in the initial $500 \mathrm{msec}$ and most responses were observed
Table 1

Frequency Count for Each 100-msec Bin in Experiment 1

\begin{tabular}{ccc}
\hline Bin & Target in First Look & Target in Second Look \\
\hline $0-99$ & 0 & 0
\end{tabular}

100-199

200-299

300-399

400-499

500-599

600-699

700-799

800-899

900-999

1,000-1,099

$1,100-1,199$

$1,200-1,299$

1,300-1,399

$1,400-1,499$

$1,500-1,599$

$1,600-1,699$

$1,700-1,799$

$1,800-1,899$

$1,900-1,999$

2,000-2,099

2,100-2,199

2,200-2,299

2,300-2,399

$2,400-2,499$

$2,500-2,599$

$2,600-2,699$

$2,700-2,799$

2,800-2,899

2,900-2,999

3,000-3,099

$3,100-3,199$

$3,200-3,299$

$3,300-3,399$

$3,400-3,499$

$3,500-3,599$

$3,600-3,699$

$3,700-3,799$

$3,800-3,899$

3,900-3,999

4,000-4,099

$4,100-4,199$

4,200-4,299

$4,300-4,399$

$4,400-4,499$

$4,500-4,599$

$4,600-4,699$

$4,700-4,799$

$4,800-4,899$

4,900-4,999

$5,000-5,099$

$5,100-5,199$

$5,200-5,299$

$5,300-5,399$

$5,400-5,499$

$5,500-5,599$

$5,600-5,699$

$5,700-5,799$

$5,800-5,899$

5,900-5,999

in the second half of the epoch (between $500 \mathrm{msec}$ and $1000 \mathrm{msec}$ after the onset of the search display). Here, $18.5 \%$ of all responses in this condition overall were recorded in this interval. Not surprisingly, the RT distribution for the target-in-second-look condition $(n=25)$ for 

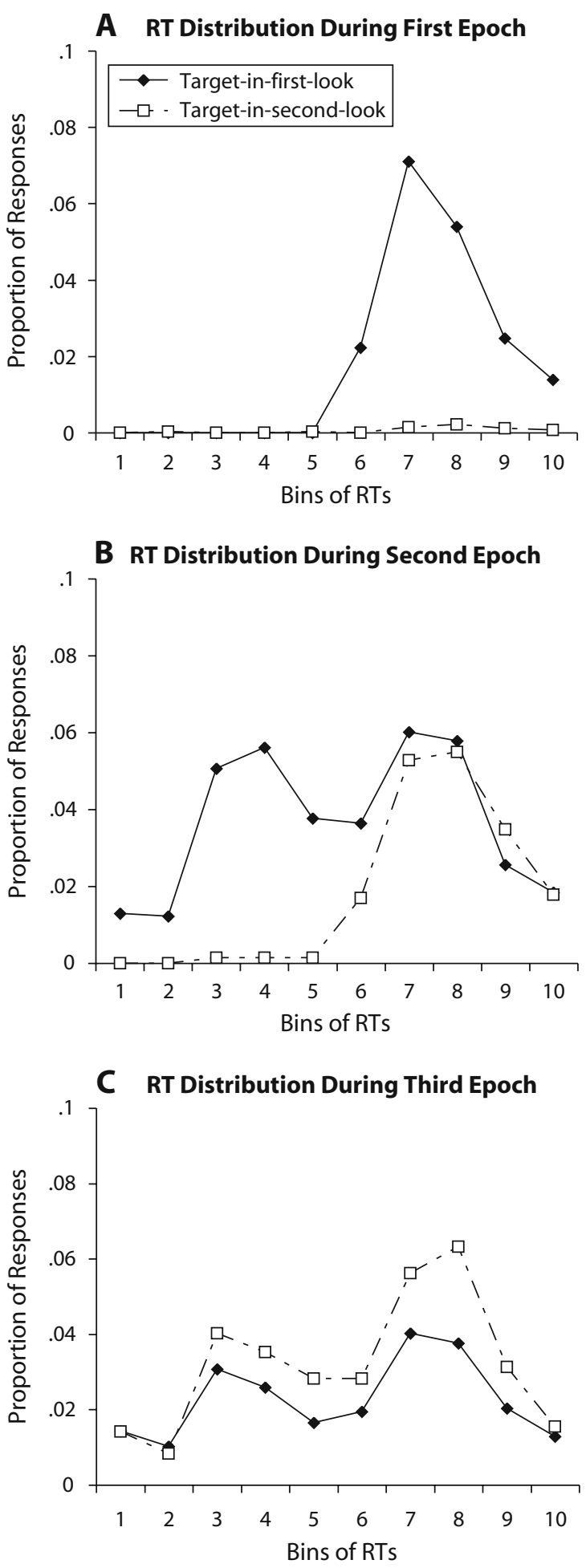

Figure 2. Correct RT distributions for Experiment 1. The data are grouped in ten 100-msec bins reflecting the length of one epoch. The search display was visible during Bin 1 and absent during Bins 2 through 9. Proportion of responses on a given bin is calculated as the number of responses on that bin divided by the total number of correct trials on that experimental condition. Full line represents the target-in-first-look condition and the slashed line represents the target-in-second-look condition. Panels A, B, and $C$ present RT distributions for both conditions in the first, second and third epochs, respectively. the first epoch shows very few responses $(0.6 \%$ of all responses in this condition). Note that these are all "lucky guesses" since the target has not yet been presented to the participants at this point.

Panel B shows RT distributions for the second epoch (i.e., following the second look at the display). Remember than in this epoch, the target has been presented twice in the target-in-first-look condition, whereas it has only been presented once in the target-in-second-look condition. The RT distribution for target in first look $(n=1,535)$ shows a typical rapid resumption effect (see Lleras et al., 2005): Forty-six percent of the responses recorded during this epoch were recorded in the first five bins of the RT distribution (0-500 msec after the onset of the search display). In contrast, the RT distribution for target in second look $(n=741)$ shows almost no responses in this interval $(0.4 \%$ of all responses in this condition and $2.4 \%$ of responses in this epoch). Clearly, rapid resumption did not occur in this condition.

Panel C shows RT distributions for the third epoch (i.e., following the third look at the display). Early-phase RTs are now observed in both conditions and to comparable extents. For the target-in-first-look condition $(n=953)$, $42.8 \%$ of the total responses in this epoch were observed in the 0 - to 500 -msec interval. For the target-in-second-look condition $(n=1,308), 39.3 \%$ of the total number of RTs in the epoch are observed in this interval. Both distributions therefore contain many early-phase RTs. The overall lower number of responses in the target-in-first-look condition is simply due to the fact that more responses were recorded in the first two epochs of this condition than in the first two epochs of the target-in-second-look condition.

\section{Discussion}

The goal of Experiment 1 was to test whether rapid resumption is dependent on preprocessing directly related to the target item. If some of the preprocessing is related to more general aspects of the display, perhaps similar to a type of visual marking, then early-phase RTs ought to be observed in the second epoch of the target-in-second-look condition. However, the RT distribution in panel B of Figure 1 clearly shows the absence of early-phase RTs in this second epoch. Thus, rapid resumption seems to be dependent on preprocessing specific to the target item. Further support for this is the finding that early-phase RTs were observed on both target-in-first-look and target-in-secondlook conditions, once participants had a minimum of two looks at a display containing the target. These results differentiate rapid resumption and visual marking (Watson \& Humphreys, 1997) in two ways. First, whereas previewing distractors in the visual marking paradigm reduces RT slopes, it does not cause the entire RT distribution to shift into a region where the average correct response occurs in 200-300 msec. Second, allowing observers to see all of the distractors prior to the presentation of the target did not in itself lead to rapid resumption, whereas it would enable visual marking. Thus, something other than the wellknown visual marking effect is at play here.

In summary, Experiment 1 provides a strong indication that rapid resumption is related to target preprocessing. 
However, we do not know to what extent it is also dependent on preprocessing of the rest of the display. For instance, rapid resumption may require the preprocessing of information not only about the target, but also about the surrounding distractor items, or perhaps even the entire layout. Experiment 2 tested for the existence of such contributions.

\section{EXPERIMENT 2}

Lleras et al. (2005) tested the contribution of display configuration or layout on the rapid resumption effect. In their Experiment 6, the identity of all search items was randomly shuffled between looks, while keeping the overall configuration constant (item identity was shuffled but all display locations remained occupied). No rapid resumption was observed. This was taken as evidence that knowledge of the scene layout was insufficient to cause rapid resumption. However, in that experiment, the target also randomly switched locations. So, any target preprocessing done during one look would not be beneficial to target processing on the subsequent look.

In the present experiment, we tried a different approach. Rather than reshuffling the identity of all the items, we changed the locations of the distractors, while leaving the target item untouched. In other words, the display configuration changed randomly from look to look, with only the target item remaining at the same location. If rapid resumption were dependent on the preprocessing of nontarget items, then it should not be observed. But if rapid resumption were solely dependent on the preprocessing of the target item, it ought to remain.

\section{Method}

Methods were identical to those of the target-in-first-look condition from Experiment 1, except that the locations of all nontarget items were randomly changed from look to look. The location and shape of the target T remained the same throughout each trial. Fifteen new participants completed 10 blocks of 64 trials.

\section{Results}

Table 2 shows the raw frequency of correct responses in Experiment 2. The normalized RT distributions for the first epoch and for the following 5 epochs (Epochs 2-6) are shown in Figure 3, along with data from Experiment 1 (target in first look) as a comparison. The first-epoch distributions (panel A) are very similar, with a tendency for slightly more responses in Experiment 2 than in the control condition data from Experiment $1\left(\chi^{2}=25.34\right.$, critical value $=9.48$, Cramer's $V=0.10$ ). The distribution ( $n=1,961$ observations) begins to move toward its dominant peak in the 500- to 600 -msec period and is positively skewed, as is typically observed in the first epoch of interrupted search experiments (e.g., Lleras et al., 2005).

The distributions for the subsequent epochs (Epochs 2-6) are shown in panel B for the control data from Experiment 1 (solid squares), and for the experimental condition in Experiment 2 in which all nontarget items keep moving to new locations (open squares). Whereas $46 \%$ of responses in Experiment 1 occurred before the half-
Table 2

Frequency Count for Each 100-msec Bin in Experiment 2

\begin{tabular}{|c|c|}
\hline Bin & Distractors Shuffled Condition \\
\hline $0-99$ & 0 \\
\hline $100-199$ & 2 \\
\hline 200-299 & 49 \\
\hline $300-399$ & 51 \\
\hline $400-499$ & 98 \\
\hline $500-599$ & 345 \\
\hline $600-699$ & 617 \\
\hline $700-799$ & 423 \\
\hline $800-899$ & 228 \\
\hline $900-999$ & 148 \\
\hline $1,000-1,099$ & 100 \\
\hline $1,100-1,199$ & 80 \\
\hline $1,200-1,299$ & 275 \\
\hline $1,300-1,399$ & 252 \\
\hline $1,400-1,499$ & 259 \\
\hline $1,500-1,599$ & 298 \\
\hline $1,600-1,699$ & 383 \\
\hline $1,700-1,799$ & 279 \\
\hline $1,800-1,899$ & 166 \\
\hline $1,900-1,999$ & 98 \\
\hline $2,000-2,099$ & 68 \\
\hline $2,100-2,199$ & 74 \\
\hline $2,200-2,299$ & 129 \\
\hline $2,300-2,399$ & 168 \\
\hline $2,400-2,499$ & 136 \\
\hline $2,500-2,599$ & 174 \\
\hline $2,600-2,699$ & 254 \\
\hline $2,700-2,799$ & 177 \\
\hline $2,800-2,899$ & 125 \\
\hline $2,900-2,999$ & 80 \\
\hline $3,000-3,099$ & 48 \\
\hline $3,100-3,199$ & 47 \\
\hline $3,200-3,299$ & 107 \\
\hline $3,300-3,399$ & 90 \\
\hline $3,400-3,499$ & 79 \\
\hline $3,500-3,599$ & 112 \\
\hline $3,600-3,699$ & 159 \\
\hline $3,700-3,799$ & 109 \\
\hline $3,800-3,899$ & 100 \\
\hline $3,900-3,999$ & 56 \\
\hline $4,000-4,099$ & 40 \\
\hline $4,100-4,199$ & 29 \\
\hline $4,200-4,299$ & 64 \\
\hline $4,300-4,399$ & 67 \\
\hline $4,400-4,499$ & 52 \\
\hline $4,500-4,599$ & 50 \\
\hline $4,600-4,699$ & 90 \\
\hline $4,700-4,799$ & 68 \\
\hline $4,800-4,899$ & 52 \\
\hline $4,900-4,999$ & 33 \\
\hline $5,000-5,099$ & 25 \\
\hline $5,100-5,199$ & 21 \\
\hline $5,200-5,299$ & 30 \\
\hline $5,300-5,399$ & 27 \\
\hline $5,400-5,499$ & 19 \\
\hline $5,500-5,599$ & 29 \\
\hline $5,600-5,699$ & 47 \\
\hline $5,700-5,799$ & 48 \\
\hline $5,800-5,899$ & 32 \\
\hline $5,900-5,999$ & 17 \\
\hline
\end{tabular}

way point of each epoch (i.e., within $500 \mathrm{msec}$ of display onset), $42 \%$ of responses in Experiment 2 did the same. This means that most of the rapid resumption effect was found even when all nontarget items changed locations $(n=5,322)$. The difference between $46 \%$ and $42 \%$ was 

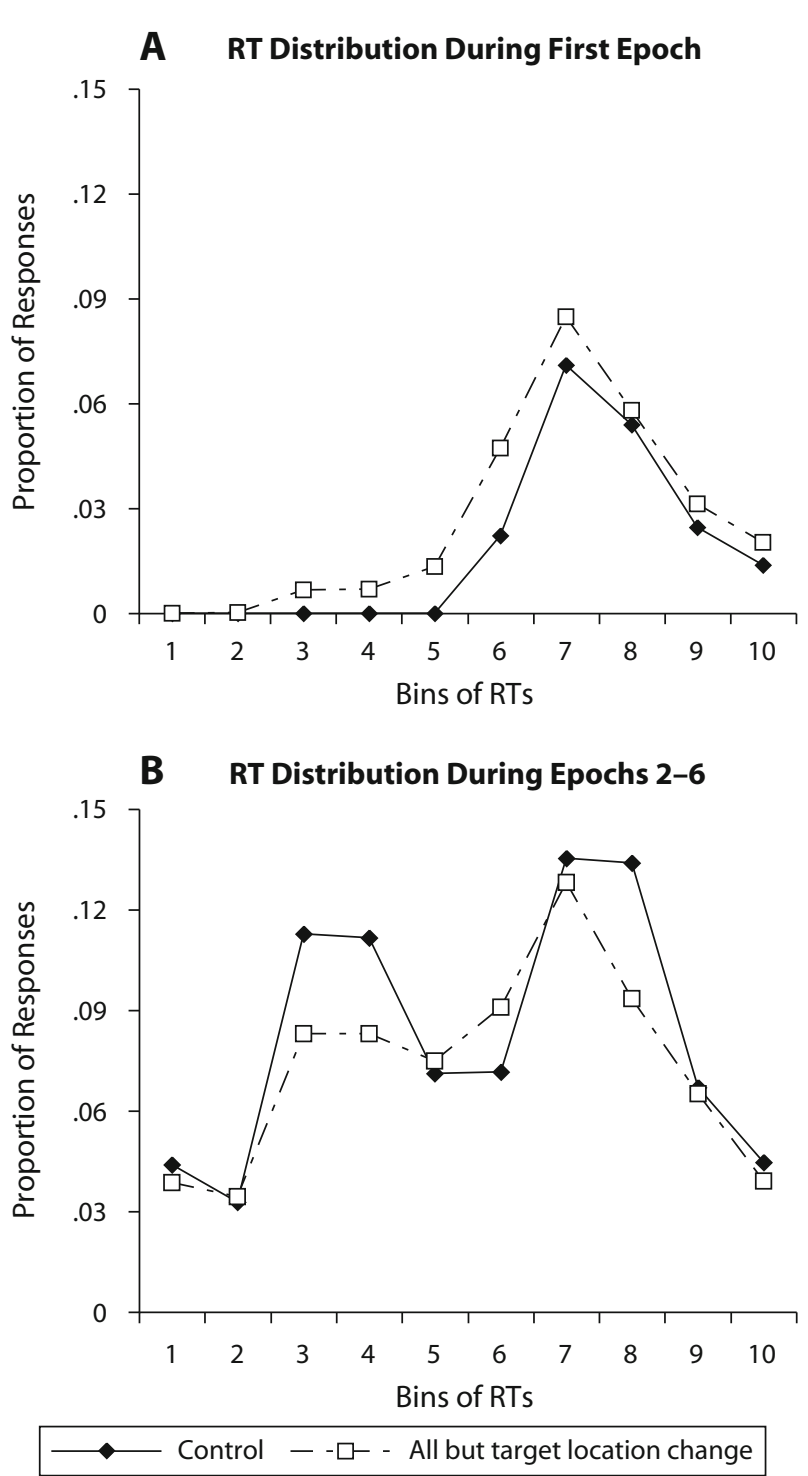

Figure 3. Correct RT distributions for Experiment 2 (slashed line) and a control condition for comparison (target present in first display condition of Experiment 1). Panel A: RT distribution during first epoch. Panel B: RT distribution during Epochs 2-6.

significant $\left(\chi^{2}=72.72\right.$, critical value $=16.92$, Cramer's $V=0.09$ ), suggesting that a small part of rapid resumption may be due to items surrounding the target. However, the level of association between the control and experimental conditions both in the first epoch (panel A) and in subsequent epochs (panel B) was still comparable (Cramer's $V=0.10$ and 0.09 , respectively).

\section{Discussion}

Experiment 2 evaluated the contribution of display-wide preprocessing to rapid resumption. When distractor items switched locations on each display presentation, participants were still able to complete the search task at levels comparable to the control condition. In fact, the mean RT in the control condition when items did not change loca- tions was $2,327 \mathrm{msec}$, whereas when distractors changed location randomly it was $2,256 \mathrm{msec}$, which was not a significant difference $[t(28)=0.17, p>.05]$. Even when distractors switched locations, early-phase responses were still obtained, although at a relatively reduced rate. (In Epoch 2 of Experiment 1, this was 46\%; in Lleras et al., 2005 , typical rates were around $52 \%$, whereas in the present experiment, the rate was about $42 \%$ ). This small reduction in early-phase RTs when items surrounding the target location are changed has also been documented elsewhere (Brady, Jungé, \& Chun, 2006; Jungé, Brady, \& Chun, 2006). Still, the fact that much of the early phase observed in Experiment 2 in spite of such large changes can be taken as strong evidence that rapid resumption is mostly related to target-specific preprocessing. Experiments 3 and 4 were designed to test what specific aspects of target preprocessing are responsible for rapid resumption.

\section{EXPERIMENTS 3 AND 4}

Taken together, the results so far suggest that in order to observe rapid resumption, participants must preprocess some information about the target (Experiment 1). They also suggest that preprocessing of other aspects of the display (e.g., parsing of the spatial configuration of the search display, costs related to starting the search task) is not involved (Experiment 2). However, this does not tell us what specific information about the target affords these early-phase RTs.

In Experiments 3 and 4, we investigated whether changes to task-relevant features of the target affected rapid resumption. The logic was as follows: if preprocessing of a relevant target attribute is required for rapid resumption, changing that attribute between successive looks should eliminate the effect. But if preprocessing of that attribute is not necessary, then changing it between successive looks should have no effect.

Experiments 3 and 4 had a nearly identical design: Within any given presentation of the search display, there was a $50 \%$ chance that the target on view was identical to the target on the previous look and a $50 \%$ chance that it was of a different color. The orientation of the target did not change between looks. The critical difference between the two experiments was that in Experiment 3, color was irrelevant to the task (participants responded to the orientation of the target T), whereas in Experiment 4, it was relevant (participants reported the color of the target T). ${ }^{2}$

\section{Method}

The methods were identical to the target-in-first-look condition of Experiment 1 and to each other, except where noted.

Tasks. Although the distractor items (Ls) were still randomly presented in one of four possible orientations (upright, upside down, pointing right or pointing left) and one of two colors (red, blue) the target item $(\mathrm{T})$ was shown only in two possible orientations (pointing right, pointing left) and two colors (red, blue), permitting participants to respond to either the orientation (Experiment 3) or the color (Experiment 4) of the T. In Experiment 3, participants used the " $z$ " key to indicate left pointing targets and the "/" key to indicate right 
pointing targets. In Experiment 4, they used the " $z$ " key to indicate blue targets and the "/" key to indicate red targets. Participants in both experiments were encouraged to respond as quickly as possible, while maintaining a level of accuracy above $95 \%$.

Design. Both experiments involved a one-factor within-subjects design. The independent variable was target change, which had two levels referring to whether the target was of the same color in the last two looks (no change) or whether the target was of different colors (color change). On every presentation of a display, there was a $50 \%$ chance that the target would change colors or remain unchanged with respect to the immediately preceding look. Fifteen new and different participants completed 10 blocks of 64 trials in each experiment.

\section{Results}

Experiment 3: Task-irrelevant change. Participants performed the interrupted search task and reported the orientation of the target $\mathrm{T}$ (right vs. left). Overall accuracy in this task was $96.3 \%$. Importantly, accuracy was unaffected by irrelevant target change $[t(14)=1.18, p>.05]$, with $96.6 \%$ accuracy on trials where the last two instances of the target were different from each other (target change condition) and $96.1 \%$ accuracy on trials where the last two instances were identical (no-target-change condition). Thus, changing task-irrelevant attributes of the target did not result in more mistakes.

Table 3 shows the raw frequency of correct responses in Experiment 3 for each of the two experimental conditions. The focus of the RT analyses was to examine differences in RT distributions in Epochs 2 and beyond. First, we calculated the average RT in this task in the two change conditions (after excluding RTs in the first epoch, where the target has only been seen once). RTs were in fact slightly faster in the change condition, mean RT of 2,477 msec, than in the no change condition, mean RT of $2,518 \mathrm{msec}$, a significant difference of $41 \mathrm{msec}[t(14)=2.80, p<$ $.05]$. In conjunction with the accuracy data, these results suggest that, if anything, performance was slightly better in the change condition than in the no change condition. There seemed to be no observable cost to changing a task-irrelevant attribute of the target between looks at the display.

Next, we performed a distribution analysis for correct RTs in Epochs 2-6 (fewer than 2.1\% of RTs were longer than $6 \mathrm{sec}$ ). Figure 4A shows the distributions for these epochs (target change $n=3,437$; no target change $n=3,457)$. As the figure suggests, the two RT distributions were not significantly different $\left(\chi^{2}=14.26\right.$, critical value $=16.92$, Cramer's $V=0.04)$. In other words, the distribution of RTs was identical regardless of whether the target had same or different colors in the last two looks prior to response. Thus, randomly changing the target color between looks has little or no effect on performance, provided that color is irrelevant to the target identification task.

Experiment 4: Task-relevant change. Participants performed the interrupted search task and reported the color of the target T (blue vs. red). Again, everything in this experiment (design, items, apparatus) was identical to Experiment 3, except that participants now reported the color rather than the orientation of the target. In both
Table 3

Frequency Count for Each 100-msec Bin in Experiment 3

\begin{tabular}{|c|c|c|}
\hline Bin & No Target Change & Target Change \\
\hline $0-99$ & 0 & - \\
\hline $100-199$ & 0 & - \\
\hline $200-299$ & 4 & - \\
\hline $300-399$ & 9 & - \\
\hline $400-499$ & 181 & - \\
\hline $500-599$ & 577 & - \\
\hline $600-699$ & 668 & - \\
\hline $700-799$ & 385 & - \\
\hline $800-899$ & 190 & - \\
\hline $900-999$ & 110 & - \\
\hline $1,000-1,099$ & 52 & 62 \\
\hline $1,100-1,199$ & 84 & 72 \\
\hline $1,200-1,299$ & 275 & 268 \\
\hline $1,300-1,399$ & 193 & 215 \\
\hline $1,400-1,499$ & 205 & 245 \\
\hline $1,500-1,599$ & 291 & 293 \\
\hline $1,600-1,699$ & 239 & 260 \\
\hline $1,700-1,799$ & 132 & 126 \\
\hline $1,800-1,899$ & 78 & 51 \\
\hline $1,900-1,999$ & 33 & 45 \\
\hline $2,000-2,099$ & 36 & 32 \\
\hline $2,100-2,199$ & 47 & 42 \\
\hline $2,200-2,299$ & 150 & 120 \\
\hline $2,300-2,399$ & 123 & 112 \\
\hline $2,400-2,499$ & 134 & 143 \\
\hline $2,500-2,599$ & 197 & 195 \\
\hline $2,600-2,699$ & 153 & 161 \\
\hline $2,700-2,799$ & 87 & 92 \\
\hline $2,800-2,899$ & 38 & 39 \\
\hline $2,900-2,999$ & 26 & 17 \\
\hline $3,000-3,099$ & 15 & 21 \\
\hline $3,100-3,199$ & 29 & 27 \\
\hline $3,200-3,299$ & 86 & 72 \\
\hline $3,300-3,399$ & 53 & 55 \\
\hline $3,400-3,499$ & 75 & 64 \\
\hline $3,500-3,599$ & 88 & 111 \\
\hline $3,600-3,699$ & 92 & 93 \\
\hline $3,700-3,799$ & 58 & 36 \\
\hline $3,800-3,899$ & 16 & 18 \\
\hline $3,900-3,999$ & 13 & 10 \\
\hline $4,000-4,099$ & 11 & 7 \\
\hline $4,100-4,199$ & 14 & 9 \\
\hline $4,200-4,299$ & 45 & 34 \\
\hline $4,300-4,399$ & 30 & 25 \\
\hline $4,400-4,499$ & 34 & 36 \\
\hline $4,500-4,599$ & 38 & 50 \\
\hline $4,600-4,699$ & 34 & 36 \\
\hline $4,700-4,799$ & 20 & 27 \\
\hline $4,800-4,899$ & 11 & 6 \\
\hline $4,900-4,999$ & 6 & 2 \\
\hline $5,000-5,099$ & 6 & 7 \\
\hline $5,100-5,199$ & 6 & 7 \\
\hline $5,200-5,299$ & 22 & 10 \\
\hline $5,300-5,399$ & 12 & 16 \\
\hline $5,400-5,499$ & 16 & 13 \\
\hline $5,500-5,599$ & 24 & 23 \\
\hline $5,600-5,699$ & 9 & 16 \\
\hline $5,700-5,799$ & 17 & 8 \\
\hline $5,800-5,899$ & 2 & 6 \\
\hline $5,900-5,999$ & 2 & 2 \\
\hline
\end{tabular}

experiments, color was the "changing" attribute of the target.

Overall accuracy here was $80.9 \%$, significantly lower than in Experiment $3[t(28)=-6.70, p<.005]$. Unlike Experiment 3, accuracy in the no-target-change condition 

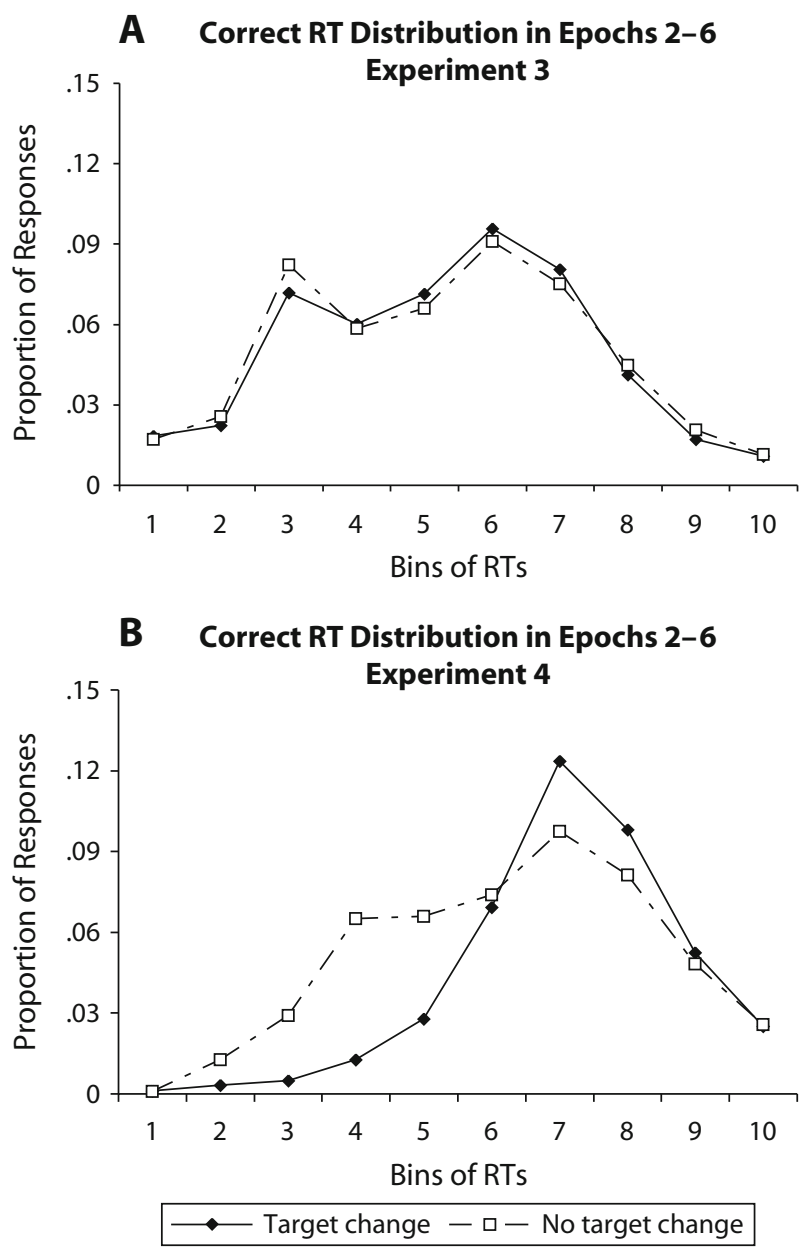

Figure 4. Correct RT distribution for Experiment 3 (panel A) and Experiment 4 (panel B) during Epochs 2-6. The full line represents the target-change condition and the slashed line represents the no-target-change condition.

was significantly higher $(88.6 \%)$ than in the target change condition $(73.3 \%)[t(14)=6.17, p<.05]$. Furthermore, excluding responses made in the first epoch, the trend in correct RTs was opposite to that of Experiment 3: RTs in the no-target-change condition (mean RT of 2,765 msec) were $48 \mathrm{msec}$ faster than in the target change condition (mean RT of 2,813 msec), though this difference did not reach significance $[t(14)=1.69, p>.05]$. Taken together, the RT and accuracy data suggest that there was a performance cost to correctly reporting a target identity if this identity was different in the previous look Next, we performed a distribution analysis for RTs in Epochs 2-6 (fewer than $3.1 \%$ of correct RTs were longer than $6,000 \mathrm{msec}$ ). Note that the raw frequency of correct responses in Experiment 4 for each of the two experimental conditions is presented in Table 4. Figure 4B shows the RT distributions for correct responses during these epochs in Experiment 4 (target change $n=2,627$; no target change $n=3,142$ ). As is evident from the figure, the two RT distributions are statistically different $\left(\chi^{2}=453.77\right.$, critical value $=16.92$, Cramer's $V=0.28$ ). In contrast to
Experiment 3, the responses in the target change condition are no longer occurring nearly as frequently in the early phase of the epoch. Also important is that the distribution in the 500 - to 1,000 -msec range is no different than the distribution observed in the first epoch of Experiment 2 $\left(\chi^{2}=8.37\right.$, critical value $=9.48$, Cramer's $\left.V=0.04\right)$. In contrast, the distribution in the no-target-change condition still showed a large proportion of early-phase RTs, though their rate $34.7 \%$ of RTs within the first $500 \mathrm{msec}$ of the epochs) was somewhat lower than what has been observed in previous experiments.

Last, because of the relatively high number of error trials, it is worthwhile to analyze the distribution of erroneous RTs in Epochs 2-6 in these two conditions. Figure 5 shows the respective distributions of errors in the notarget-change condition (dotted line, $n=472$ ) and target change condition (full line, $n=1,021$ ). Note that the scale has now changed (about one fifth of the amplitude of the scales in Figure 3 displaying correct RTs). As the figure suggests, these two distributions differ reliably from each other $\left(\chi^{2}=96.03\right.$, critical value $=16.91$, Cramer's $V=$ 0.253 ). Notably, most of the errors committed in the target change condition occurred in the first $500 \mathrm{msec}$ of the epochs $(61 \%)$, and the error rate peaked between 200 and $300 \mathrm{msec}$, whereas the errors committed in the no-targetchange condition were more evenly distributed across the epoch $(49.3 \%$ in the first half of the epoch) and peaked between 500 and $600 \mathrm{msec}$. This implies that when participants where responding erroneously, they tended to do so primarily on change trials and they did so during the time period when this would have resulted in early-phase RTs, had the target item color not changed from the previous look. The similarity of the changed-color target to the original target in its other characteristics (i.e., location, orientation) must have been sufficiently high on these trials to trigger a false alarm based on the preprocessed color information.

\section{Discussion}

Experiments 3 and 4 assessed how changes to the target between looks affected rapid resumption. Crucially, the changing feature - target color - was the same across the two experiments. The only difference was that, in Experiment 3, participants reported the orientation of the target, making the feature of color irrelevant to the task, whereas in Experiment 4 they reported the color of the target, making it task relevant.

The results of Experiment 3 showed that a task-irrelevant color change had little influence on rapid resumption, whereas the results of Experiment 4 showed that a taskrelevant color change eliminated it altogether, even reducing it somewhat on those trials when the target did not change colors. Together, these results suggest that target preprocessing involves something more than a simple measure of what distinguishes the target from the distractors (i.e., how much an item resembles a T). If this were all then a color change would never influence the results, since color does not distinguish the target from the distractors. Instead, a color change alters the results only 
Table 4

Frequency Count for Each 100-msec Bin in Experiment 4

\begin{tabular}{|c|c|c|}
\hline Bin & No Target Change & Target Change \\
\hline $0-99$ & 0 & - \\
\hline $100-199$ & 0 & - \\
\hline $200-299$ & 2 & - \\
\hline $300-399$ & 3 & - \\
\hline $400-499$ & 21 & - \\
\hline $500-599$ & 258 & - \\
\hline $600-699$ & 601 & - \\
\hline $700-799$ & 511 & - \\
\hline $800-899$ & 275 & - \\
\hline $900-999$ & 133 & - \\
\hline $1,000-1,099$ & 2 & 0 \\
\hline $1,100-1,199$ & 28 & 11 \\
\hline $1,200-1,299$ & 95 & 10 \\
\hline $1,300-1,399$ & 210 & 27 \\
\hline $1,400-1,499$ & 183 & 72 \\
\hline $1,500-1,599$ & 235 & 227 \\
\hline $1,600-1,699$ & 277 & 363 \\
\hline $1,700-1,799$ & 219 & 252 \\
\hline $1,800-1,899$ & 118 & 142 \\
\hline $1,900-1,999$ & 64 & 83 \\
\hline $2,000-2,099$ & 2 & 1 \\
\hline $2,100-2,199$ & 24 & 2 \\
\hline $2,200-2,299$ & 38 & 12 \\
\hline $2,300-2,399$ & 103 & 23 \\
\hline $2,400-2,499$ & 119 & 44 \\
\hline $2,500-2,599$ & 123 & 111 \\
\hline $2,600-2,699$ & 168 & 210 \\
\hline $2,700-2,799$ & 149 & 183 \\
\hline $2,800-2,899$ & 91 & 96 \\
\hline $2,900-2,999$ & 40 & 34 \\
\hline $3,000-3,099$ & 1 & 2 \\
\hline $3,100-3,199$ & 15 & 2 \\
\hline $3,200-3,299$ & 29 & 3 \\
\hline $3,300-3,399$ & 62 & 14 \\
\hline $3,400-3,499$ & 55 & 37 \\
\hline $3,500-3,599$ & 66 & 60 \\
\hline $3,600-3,699$ & 101 & 123 \\
\hline $3,700-3,799$ & 75 & 97 \\
\hline $3,800-3,899$ & 60 & 51 \\
\hline $3,900-3,999$ & 28 & 23 \\
\hline $4,000-4,099$ & 0 & 1 \\
\hline $4,100-4,199$ & 8 & 4 \\
\hline $4,200-4,299$ & 16 & 2 \\
\hline $4,300-4,399$ & 22 & 12 \\
\hline $4,400-4,499$ & 33 & 18 \\
\hline $4,500-4,599$ & 23 & 25 \\
\hline $4,600-4,699$ & 42 & 58 \\
\hline $4,700-4,799$ & 39 & 57 \\
\hline $4,800-4,899$ & 21 & 28 \\
\hline $4,900-4,999$ & 18 & 10 \\
\hline $5,000-5,099$ & 0 & 3 \\
\hline $5,100-5,199$ & 5 & 1 \\
\hline $5,200-5,299$ & 5 & 3 \\
\hline $5,300-5,399$ & 12 & 4 \\
\hline $5,400-5,499$ & 24 & 4 \\
\hline $5,500-5,599$ & 17 & 12 \\
\hline $5,600-5,699$ & 25 & 23 \\
\hline $5,700-5,799$ & 28 & 28 \\
\hline $5,800-5,899$ & 13 & 12 \\
\hline $5,900-5,999$ & 11 & 7 \\
\hline
\end{tabular}

when it is relevant to the response that must ultimately be made when the target is identified.

An alternative interpretation is that rather than eliminating rapid resumption in the change condition of Ex- periment 4 , responses were simply delayed due to response competition. For example, if one assumes that the response-relevant attribute of the target was included in the preprocessing of the target in the next-to-last look at the display, one might expect a response competition effect on the last look, when the newly changed target activates a different response. Although such a response competition account remains a possibility, we think it is implausible for two reasons. First, the reaction time distribution in the 500- to $1,000-\mathrm{msec}$ portion of the change condition in Figure 3B is statistically identical to the first epoch distribution of Experiment 2 (Figure 2A). In other words, the RT distribution looks identical to other RT distributions in which RR is not observed and in which there is no opportunity for response competition to occur (see also the RT distribution of the first epoch in all of the present experiments, as well as Epoch 2 in the target-in-second look condition of Experiment 1). Since there is no need to invoke response competition to explain those results, it seems unnecessary to invoke it for these results.

A second reason for doubting the response competition account relates to the magnitude of such effect, had it been present in our data. Typically, response competition effects are in the order of tens of milliseconds (10-20 or even as much as $50 \mathrm{msec}$ ); we are unaware of any response competition effects in the literature with magnitudes larger than $100 \mathrm{msec}$. Yet a quick inspection of Figure 3B reveals that if response competition was the underlying cause for the rightward shift of many responses in the change-RT distribution, the magnitude of such an effect would have had to be in the order of 300 to $400 \mathrm{msec}$. Therefore, we feel confident that the absence of a large number of earlyphase responses in the change condition of Experiment 4 does not reflect a response-competition rightward shift of the data, but rather an overall absence of rapid resumption responses.

In sum, these results show that target preprocessing goes beyond an analysis of the distinguishing features of the target (here, a T vs. an L junction). However, at the same time, they show that preprocessing does not automatically extend to every feature of the target (here, color and orientation). Indeed, only changes made to response-relevant features of the target (color in Experiment 4) interfered with performance. The preprocessing of the target must therefore include at least those features relevant to the task.

Last, it is also worth comparing the results of Experiment 4 here to the results of Experiment 5 in Lleras et al. (2005). In that experiment, participants were at chance if the interrupted search task stopped abruptly after just one epoch, even though they demonstrated a large rapid resumption effect when the interrupted search task continued after the first epoch. Those results suggested that participants were generally unaware of whatever target preprocessing they had done. Here, in the present Experiment 4, the analysis of the error data (Figure 3C) suggests that this unconsciously preprocessed information can lead to a false positive response, provided that the new display contains an item similar to the perceptual hypothesis in other respects (i.e., location, shape, orientation). 


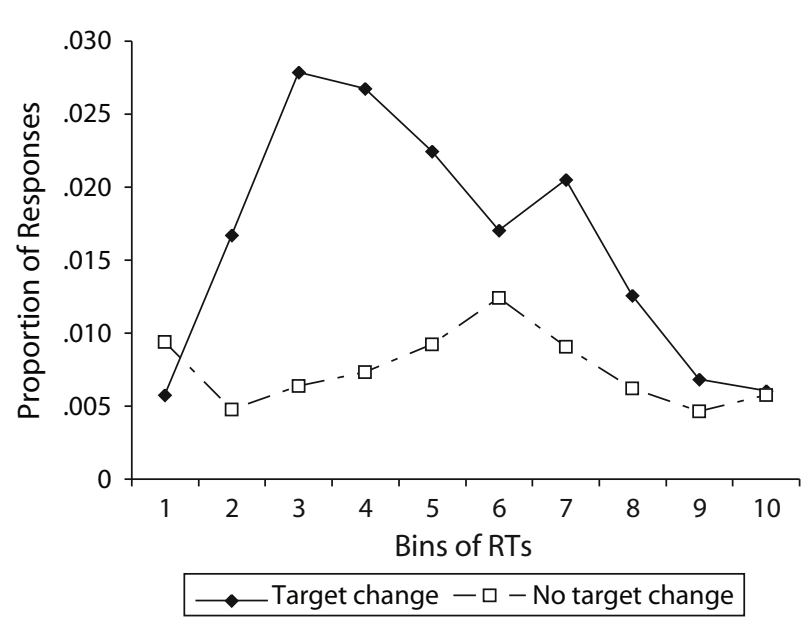

Figure 5. RT distributions for incorrect trials in Experiment 4 for both target change (full line) and no-target-change (slashed line) conditions during Epochs 2-6. Note the change in scale.

\section{GENERAL DISCUSSION}

The aim of this study was to examine in greater detail the rapid resumption effect first described in Lleras et al. (2005). Specifically, we tested the claim that the preprocessing responsible for the fast responses in interrupted search tasks (early-phase RTs) involves information specific to the target (Lleras et al., 2005). We also explored the extent to which the preprocessing contains detailed feature information about target features.

In Experiments 1 and 2, we found that preprocessing of target-specific information was necessary and largely sufficient to obtain rapid resumption. When the target item was made unavailable to the participants during a first look, rapid resumption was not observed in the following epoch (Experiment 1). Similarly, when all items except the target randomly changed locations, rapid resumption was still observed (Experiment 2). Experiments 3 and 4 showed that changes to a response-relevant feature eliminated rapid resumption, whereas changes to task-irrelevant features did not. This implied that perceptual preprocessing included response-defining information (color) in addition to the target-defining information (shape). Response-irrelevant information (orientation) does not seem to be extracted in the perceptual preprocessing.

\section{Reentrant Processing Theory}

Lleras et al. (2005) originally interpreted rapid resumption within the framework of a reentrant processing theory of visual awareness of Di Lollo et al. (2000). According to this theory, perception is an iterative process whereby visual information is processed at various levels, most notably a higher level associated with object representations and a lower level associated with precategorical sensory input. Perceptual awareness is achieved once a "perceptual hypothesis" about a candidate object is created and confirmed against the current sensory input. Importantly, observers do not form conscious pictures of perceptual hypotheses that fail to be confirmed, which can happen when sensory information regarding the initial item is no longer present in the visual system, as is the case in visual backward masking. ${ }^{3}$

Within this context, Lleras et al. (2005) proposed that rapid resumption occurs as a relative benefit of having already created a perceptual hypothesis about the target (based on the first look) prior to the appearance of the second look necessary for perceptual confirmation. To illustrate in greater detail, refer to the last look at a display as look $L$. The authors proposed that on trials on which rapid resumption was observed, participants had already created a perceptual hypothesis about the target on look $L-1$. But, because sensory information regarding the target had been removed from the display before this hypothesis could be confirmed, participants did not become aware of the target during that epoch. In other words, the perceptual hypothesis about the target remained unconfirmed. However, upon re-presentation of the display (i.e., on look $L$ ), the partial information about the target contained in this perceptual hypothesis was combined with sensory information to complete target processing, resulting in both faster awareness of the target and faster responses than if the perceptual hypothesis had to be constructed (and confirmed) from scratch.

When viewed in this context, the findings of the present study are telling in several ways. The results from Experiment 1 suggest that some preprocessing of the targetperhaps in the form of a perceptual hypothesis - is largely responsible for rapid resumption, since it never occurs following the first look at the target. The results from Experiment 2 add to this by suggesting that failure to confirm perceptual hypotheses about the distractors is relatively benign: when distractors changed locations from one look to the next (making it impossible to confirm perceptual hypotheses about them), the rate of early-phase RTs was only slightly reduced.

More interestingly, we can interpret Experiments 3 and 4 as direct examinations of the perceptual hypotheses involved. Experiment 3 showed that random changes to the color of the target between looks did not affect the likelihood or the rate of rapid resumption. This result is inconclusive with regard to the extent to which task-irrelevant details are represented in the hypothesis. On the one hand, the failure to interfere with rapid resumption could be taken as a failure to represent the "changing" feature. On the other hand, it could simply mean that the change in feature-value did not interfere with the task at hand when it was irrelevant to the task. But what can be concluded from this experiment is that the "confirmation" or "matching" stage in the reentrant processes view concerns taskrelevant features; a precise match on all features between the perceptual hypothesis and the new sensory image was not required. This is inline with research from Hayhoe and colleagues pointing to the privileged role of task-relevant information in what is attended and remembered (Hayhoe, Bensinger, \& Ballard, 1998).

In Experiment 4, the analysis of the response error data (Figure 3C) showed a strong interference effect when the 
sensory information did not match the perceptual hypothesis along the response-feature dimension. From this we can conclude that preprocessing of the target (i.e., the perceptual hypothesis) included the information needed to make the response. If this information had not been represented, rapid resumption would have been observed (as suggested by the results of Experiment 3). It is important to remember that the changing feature was the same in Experiments 3 and 4 . In both experiments, the target could change color between looks. Whereas the results of Experiment 3 suggest that color was not represented between looks when it was not relevant to the task, the results of Experiments 4 strongly suggest that color was represented between looks when it was the response-defining feature of the target.

In summary, the results of the present study inform us as to the contents of the preprocessing that allows rapid resumption to occur. In the context of the reentrant processing view of awareness, these results are first of all consistent with the claim that rapid resumption reflects the formation of perceptual hypothesis on the basis of one look and the subsequent confirmation of that hypothesis in a subsequent look. Second, they suggest that perceptual hypotheses contain information not only about the location and shape of the target, but also about features relevant for the task at hand. Further, these results tell us that the confirmation process (the matching of the perceptual hypothesis to sensory information) is adaptive and flexible. A perfect match on all features is not required for awareness of target identity; the match only needs to be made for the features that are relevant to the response. This implies that the confirmation process either does not even represent irrelevant features in the hypothesis, or it may be robust enough to allow for mismatches along irrelevant features of the target.

\section{But Is a Reentrant Theory Necessary?}

Although we have given a reentrant account of the data from the interrupted search task, the results do not in themselves necessitate a reentrant account: they are also consistent with a strictly feedforward account, wherein which the effects of interruption are analogous to stopping a conveyer belt on an assembly line. In this account, target processing is simply halted during the enforced interruption of search, and then rejoined as rapidly as possible once the search display is returned to view.

The findings to be explained from this perspective are that sometimes halting the process allows for a rapid return to the search task (early-phase RTs), whereas at other times halting the process means the search process must begin from scratch (late-phase RTs). Let us briefly review the factors that seem to be critical in tipping the balance in favor of one outcome or the other.

Rapid resumption has been observed when the search display is interrupted with a blank screen for up to $3 \mathrm{sec}$ in duration (Lleras et al., 2005, Experiments 1 and 3), when the duration of the interruption is unpredictable (Lleras et al., 2005, Experiment 4), when the search task involves two temporally interleaved displays with different configurations (Lleras et al., 2005, Experiment 2), when there is a new configuration of distractors on each look (present Experiment 2), and when the target changes in a salient but response-irrelevant feature from look to look (present Experiment 3). All of these characteristics are easy to understand with a model based on the idea of a halted conveyer belt.

What is more difficult to do is to predict the observed failures of rapid resumption with this model. Rapid resumption does not occur when the location of the target is randomly relocated from look to look (Lleras et al., 2005, Experiment 6), nor when the prior look is a display of distractors in a stable configuration (present Experiment 1), nor when the target changes from look to look in a response-relevant feature (present Experiment 4), nor when the target location is validly precued prior to first look (Lleras et al., 2005), and not when the expected target is presented to the center of fovea (van Zoest, Lleras, Kingstone, \& Enns, in press). Furthermore, when an interruption occurs and the search display is never shown again, there is no evidence that participants have any access to the preprocessing that is demonstrably present when the display reappears (Lleras et al., 2005, Experiment 5). These findings constrain a feedforward model to include certain information about the target (its location and its response relevant attributes) but not other information about it (no explicit link to the response, little representation of its contextual surroundings). Such a model also would have to account for why the preprocessed information about the target cannot be accessed unless the display is shown again. We know of no a priori grounds on which to make these predictions.

On the other hand, all of these constraints fit very naturally into the reentrant account, namely that the participant is testing for a match between a specific hypothesis about the target and the current sensory evidence available with regard to that hypothesis. Such a hypothesis would naturally include location and response relevant information but little else. The reentrant hypothesis also has a natural account of why the preprocessed information cannot be accessed consciously, namely, the reentrant activity must match the sensory input before conscious awareness is even possible.

\section{Adaptive Resonance Theory}

It is interesting to note the similarity between the reentrant process view of awareness derived from masking research (Di Lollo et al., 2000; Moore \& Lleras, 2005) and the adaptive resonance theory (ART) derived from research on pattern recognition (Carpenter \& Grossberg, 1987, 2003; Grossberg, 1976a, 1976b). ART is a more general-purpose neural-computational model of human information processing, capable of pattern recognition and unsupervised learning. In it, sensory inputs are initially processed until they activate or produce a higher level code. The activated code (also referred to as the hypothesis) is then tested as a top-down expectation against sensory input. When a match occurs, the system enters a resonant state, which allows for learning and pattern recognition. Importantly, matches need not be exact, and only matches along "critical features" of the hypothesis are 
necessary for resonance. When a near match leads to resonance, it is said that the variations in features were within the "attentional focus" of the critical features of the task (Carpenter \& Grossberg, 2003). On the other hand, when the bottom-up input is too different from the hypothesis (or activated code), the identification process is reset and started anew. In ART, the matching criterion is determined by the parameter $\rho$, also referred to as vigilance.

It is remarkable that both in the reentrant process view and in ART very analogous language is used to describe the inner workings of both models, even though these neural-computational models have been developed with very different goals. Furthermore, ART includes the $\rho$ parameter that is used to specify the match/mismatch criterion based on the contents of the critical features of the task. It is then easy to see that analogous components could be at work in the reentrant processes view. The "attentional focus" could be determined by the most immediately critical features to the task: the target-defining feature (here, the critical line junction distinguishing a $\mathrm{T}$ from an L) and the response-defining feature (item orientation in Experiment 3 and item color in Experiment 4). Other features would then fall outside of this "attentional focus," and therefore mismatches along those dimensions would not affect the overall match of sensory input to perceptual hypothesis. In other words, changes to targets along noncritical features would not be "misperceived," nor would they interfere with the confirmation of the perceptual hypothesis. On the other hand, changes to targets along critical features would lead to a mismatch of the sensory input to the perceptual hypothesis, which would in turn lead to a delay in the awareness of and response to the target.

\section{Unconscious Motor Preparation?}

In our account of Experiment 4, we suggested that participants may not become aware of response-relevant information until the perceptual hypothesis is confirmed with new sensory information. This possibility is strongly supported by data in Lleras et al. (2005), which showed participants unable to respond correctly to a target if they had no conscious awareness of having seen it (Lleras et al., 2005). In view of the results from the present Experiment 4 , we now argue that participants must have been representing the relevant information at some level, prior to becoming aware of the target. At first, this claim might seem rather surprising, as it essentially says that the target was unconsciously identified by the participants. However, this claim is not as radical as it may appear at first. First, it is important to dissociate processes leading to the conscious identification of an item from sensory encoding processes inaccessible to conscious scrutiny. We argue that the formation of a perceptual hypothesis is the result of such unconscious processes. Furthermore, we have no evidence at this point that the response-relevant information carried by the unconfirmed perceptual hypothesis has any influence on motor control or on conscious target identification (apart from speeding this up when a matching stimulus appears). Rather, one could argue that response-relevant information was represented to some large degree in the perceptual hypothesis and that a mismatch along that feature dimension could lead to the rejection of the entire hypothesis and therefore indirectly delaying awareness and identification of the target.

On the other hand, a distinct possibility is that response-relevant information represented in the perceptual hypothesis may activate associated motor responses, before awareness of the target takes place. This possibility is truly a case of unconscious perception, as understood in the field of masked priming. In typical masked priming studies, participants are asked to view a rapid sequence of three items: a brief prime item, followed by an overlapping mask item, which is in turn followed by the target item. By manipulating the prime-mask relationship along geometric, temporal and spatial similarity factors, experimenters can find conditions in which participants never become aware of the prime identity (as indicated by chance performance in a prime identification task), yet the prime identity significantly impacts target identification processes (e.g., Eimer \& Schlaghecken, 2002; Klapp \& Hinkley, 2002; Klotz \& Neumann, 1999; Lleras \& Enns, 2004, 2005, 2006; Vorberg et al., 2003). Therefore, it is entirely likely that having represented (to some extent) response-relevant information in the yet unconfirmed perceptual hypothesis, this information may find a way of unconsciously activating associated motor responses, just as unconfirmed perceptual hypotheses seem to do in masked priming. Further studies are needed to disentangle these two possibilities.

\section{Memory for Distractors During Interrupted Visual Search}

In a set of very influential studies, Horowitz and Wolfe $(1998,2001,2003)$ provided evidence that observers show no memory benefits in a variety of visual search tasks. For example, Horowitz and Wolfe $(1998,2003)$ showed that search slopes are identical when stimuli in a visual search task are randomly relocated on the display every $100 \mathrm{msec}$ (the dynamic condition) compared to when the stimuli stay at the same location throughout the trial (the static condition). The authors argued that visual memory would be of no use to the participants in the dynamic condition (as the stimuli were constantly being relocated); in contrast, they argued that if memory information about inspected-and-rejected distractors helps observers complete their search, observers should search the display more efficiently in the static condition. Because there was in fact no difference in search efficiency between the static and dynamic conditions, the authors argued that both types of search must then arise from "amnesic" search mechanisms. In other words, Horowitz and Wolfe argued that information from rejected distractors is not stored in visual short-term memory.

Since then, a number of reports have tested this "amnesic" theory of visual search (e.g., McCarley, Wang, Kramer, Irwin, \& Peterson, 2003; Oh \& Kim, 2004; Peterson, Kramer, Wang, Irwin, \& McCarley, 2001; Rensink, 2000; Takeda \& Yagi, 2000; Woodman \& Luck, 2004). Reviewing all of these findings is out of the scope of the present report. Yet, we believe our results can add some in- 
formation to the larger debate regarding the role of memory in search, and in particular, regarding the memory processes involved in processing distractors.

Two points are worth making in this respect. First, insofar as rapid resumption is evidence that perceptual processing continues during interruptions in sensory input (i.e., while the search display is off), rapid resumption is in itself evidence of a type of memory for both target and distractor stimuli. This follows because there is no a priori reason to distinguish the perceptual role of distractors and targets prior to the detection and identification of the target. Therefore, just as we have found evidence that partial information about the target can be stored in the visual system between displays, partial information about distractors must also be stored before the target is found. To be certain, the level of analysis here is different from that of Horowitz and Wolfe $(1998,2003)$ : Whereas we are showing evidence of memory traces for distractor stimuli prior to their identification, Horowitz and Wolfe's (1998) original point was that once an observer has identified and rejected a distractor, information about that rejected distractor does not influence the remaining search process. Nevertheless, we take the occurrence of rapid resumption as evidence of a form of memory during interrupted visual search.

On a second point, which is more directly relevant to Horowitz and Wolfe's $(1998,2003)$ specific claim that memory for distractor information does not affect search performance, we believe our data suggests that they do. The reader is directed to Figure 3B, which displays the results from Experiment 2. This experiment perhaps approximates the closest some of Horowitz and Wolfe's (1998) experiments. In particular, our Experiment 2 is quite close to their dynamic condition in which items are constantly being relocated (every $100 \mathrm{msec}$ ), except that in our Experiment, relocation occurs after a 900-msec blank display and the target does not move. When we compare the rate of rapid resumption in our "dynamic" condition to that of our "static" (i.e., when distractors stay at the same location on every display), there is an observable decrease in the rate of early-phase responses. Although we did not design this experiment to directly test for this difference, this decrease is suggestive that the random relocation of distractors slows down search. In other words, unlike Horowitz and Wolfe $(1998,2003)$, we find evidence that the locations of (some) distractors were stored in memory. This somewhat tenuous conjecture has now found support in the findings from a separate group of researchers, who designed an interrupted search paradigm to directly test the contribution of memory for distractor locations on rapid resumption (Jungé et al., 2006). Jungé et al.'s results show significant decreases in the rate of rapid resumption when the location of distractors nearby the target is changed between looks. Taken together, our data and those of Jungé et al. seem to suggest that memory for distractor locations does impact the efficiency of the resumption of visual search. In this manner, our results regarding the interrupted search paradigm are consistent with similar results reported using static search and contingent search paradigms (e.g., McCarley, Wang, Kramer, Irwin, \& Pe- terson, 2003; Oh \& Kim, 2004; Peterson, Kramer, Wang, Irwin, \& McCarley, 2001; Rensink, 2000; Takeda \& Yagi, 2000; Woodman \& Luck, 2004).

\section{Contextual Cuing}

One final point concerns possible links between rapid resumption and the phenomenon of contextual cuing (e.g., Chun \& Jiang, 1998, 2003). Chun and colleagues have done extensive research to document that search performance is improved when the spatial configuration of items in a display is repeated over different trials (compared to trials in which the spatial configuration is novel). One surface similarity of this effect and rapid resumption is that observers see the same search display several times. A crucial difference, however, is that in contextual cuing, each time participants see the same search display, they find the target, whereas in rapid resumption, participants only see the same search display until they find the target. In other words, search configurations are novel for each interrupted search trial. This means that participants in a contextual cuing study have a chance to build a memory linking a display configuration with a target location for use in later trials. Any similar memory trace formed by participants in an interrupted search task would be useless, since the same displays are never repeated.

A more interesting similarity between contextual cuing and rapid resumption is the extent to which both are sensitive to changes in the configuration of distractors surrounding the target. As already noted, rapid resumption is reduced when the distractors around the target are randomly relocated and the same seems to be true of contextual cuing (Brady \& Chun, 2007; Jungé et al., 2006). More research is needed to investigate what exactly it is about the local configuration of distractors that affects search performance in this way.

\section{CONCLUSION}

Our results indicate that rapid resumption of search is due to a preprocessing of the display that is specific to the target item. Moreover, this preprocessing is based on partial target information obtained on the penultimate look at the display prior to responding. This partial information appears to include response-relevant features of the target. We propose that the preprocessing of the target reflected in rapid resumption is the formation and partial testing of perceptual hypotheses about the target. One remarkable feature of these perceptual hypotheses is that participants have little if any conscious access to the information contained in them. Yet, they work effectively to connect visual information acquired in separate glances at the same scene in a somewhat seamless fashion.

\section{AUTHOR NOTE}

This work was supported by National Science Foundation Grant 0527361 awarded to A.L., who was the leading investigator in this project. R.A.R. and J.T.E. contributed to the design of the experiments and the theoretical interpretation of the results. A.L. designed, conducted, and analyzed all four experiments, interpreted the results, and contributed to the theoretical interpretation of the results. Correspondence concerning this article should be addressed to A. Lleras, Department of Psychology 
and Beckman Institute, University of Illinois at Urbana-Champaign, 603 East Daniel St., Champaign, IL 61820 (e-mail: alleras@uiuc.edu).

\section{REFERENCES}

Brady, T. F., \& Chun, M. M. (2007). Spatial constraints on learning in visual search: Modeling contextual cuing. Journal of Experimental Psychology: Human Perception \& Performance, 33, 798-815.

Brady, T. F., Jungé, J. A., \& Chun, M. M. (2006, May). Local and global influences on hypothesis testing during rapid resumption of visual search. Poster presented at the 2006 Annual Meeting of the Vision Sciences Society, Sarasota, FL.

Carpenter, G. A., \& Grossberg, S. (1987). A massively parallel architecture for a self-organizing neural pattern recognition by machine. Computer Vision, Graphics, \& Image Processing, 37, 54-115.

Carpenter, G. A., \& GrossberG, S. (2003). Adaptive resonance theory. In M. A. Arbib (Ed.), The handbook of brain theory and neural networks (2nd ed., pp. 87-90). Cambridge, MA: MIT Press.

Chun, M. M., \& Jiang, Y. (1998). Contextual cuing: Implicit learning and memory of visual context guides spatial attention. Cognitive Psychology, 36, 28-71.

ChUn, M. M., \& JiAnG, Y. (2003). Implicit, long-term spatial contextual memory. Journal of Experimental Psychology: Learning, Memory, \& Cognition, 29, 224-234.

Di Lollo, V., Enns, J. T., \& Rensink, R. A. (2000). Competition for consciousness among visual events: The psychophysics of reentrant visual processes. Journal of Experimental Psychology: General, 129, 481-507.

Donk, M., \& Verburg, R. C. (2004). Prioritizing new elements with a brief preview period: Evidence against visual marking. Psychonomic Bulletin \& Review, 11, 282-288.

DownING, P. E. (2000). Interaction between visual working memory and selective attention. Psychological Science, 11, 467-473.

Eimer, M., \& Schlaghecken, F. (2002). Links between conscious awareness and response inhibition: Evidence from masked priming. Psychonomic Bulletin \& Review, 9, 514-520.

ENNS, J. T., \& Di Lollo, V. (2000). What's new in visual masking? Trends in Cognitive Sciences, 4, 345-352.

Fockert, J. W., Rees, G., Frith, C. D., \& Lavie, N. (2001). The role of working memory in visual selective attention. Science, 291, 18031806.

Grossberg, S. (1976a). Adaptive pattern classification and universal recoding: I. Parallel development and coding of neural feature detectors. Biological Cybernetics, 23, 121-134.

Grossberg, S. (1976b). Adaptive pattern classification and universal recoding: II. Feedback, expectation, olfaction, and illusions. Biological Cybernetics, 23, 187-202.

HAN, S. H., \& Kim, M. S. (2004). Visual search does not remain efficient when executive working memory is working. Psychological Science, 15, 623-628

Hayhoe, M. M., Bensinger, D. G., \& Ballard, D. H. (1998). Task constraints in visual working memory. Vision Research, 38, 125-137.

Horowitz, T. S., \& Wolfe, J. M. (1998). Visual search has no memory. Nature, 394, 575-577.

Horowitz, T. S., \& Wolfe, J. M. (2001). Search for multiple targets: Remember the targets, forget the search. Perception \& Psychophysics, 63, 272-285.

Horowitz, T. S., \& Wolfe, J. M. (2003). Memory for rejected distractors in visual search? Visual Cognition, 10, 257-298.

Jungé, J. A., Brady, T. F., \& Chun, M. M. (2006). The contents of perceptual hypotheses: Evidence from rapid resumption of interrupted visual search. Manuscript submitted for publication.

KLAPP, S. T., \& HinKLEY, L. B. (2002). The negative compatibility effect: Unconscious inhibition influences reaction time and response selection. Journal of Experimental Psychology: General, 131, 255269.

KLeIN, R. M., \& McInNES, W. J. (1999). Inhibition of return is a foraging facilitator in visual search. Psychological Science, 10, 790-801.

Klotz, W., \& Neumann, O. (1999). Motor activation without conscious discrimination in metacontrast masking. Journal of Experimental Psychology: Human Perception \& Performance, 25, 976-992.

Kunar, M., Humphreys, G., \& SMITH, K. J. (2003). History matters: The preview benefit in search is not onset capture. Psychological Science, 14, 181-185.

Lleras, A., \& EnNs, J. T. (2004). Negative compatibility or object updating? A cautionary tale of mask-dependent priming. Journal of Experimental Psychology: General, 133, 475-493.

LLERAS, A., \& ENNS, J. T. (2005). Updating a cautionary tale of masked priming: A reply to Klapp (2005). Journal of Experimental Psychology: General, 134, 436-440.

Lleras, A., \& ENNS, J. T. (2006). How much like a target can a mask be? Geometric, spatial, and temporal similarity in priming - A reply to Schlaghecken and Eimer. Journal of Experimental Psychology: General, 135, 495-500.

Lleras, A., Rensink, R. A., \& Enns, J. T. (2005). Rapid resumption of interrupted visual search: new insights on the interaction between vision and memory. Psychological Science, 16, 684-688.

McCarley, J. S., Wang, R. F., Kramer, A. F., Irwin, D. E., \& Peterson, M. S. (2003). How much memory does oculomotor search have? Psychological Science, 14, 422-426.

Moore, C. M., \& Lleras, A. (2005). On the role of object representations in substitution masking. Journal of Experimental Psychology: Human Perception \& Performance, 31, 1171-1180.

Он, S. H., \& Kıм, M. S. (2004). The role of spatial working memory in visual search efficiency. Psychonomic Bulletin \& Review, 11, 275-281.

Peterson, M. S., Kramer, A. F., Wang, R. F., Irwin, D. E., \& McCarley, J. S. (2001). Visual search has memory. Psychological Science, 12, 287-292.

RENSINK, R. A. (2000). Visual search for change: A probe into the nature of attentional processing. Visual Cognition, 7, 345-376.

TAKEDA, Y., \& YAGI, A. (2000). Inhibitory tagging in visual search can be found if search items remain visible. Perception \& Psychophysics, 62, 927-934.

van Zoest, W., Lleras, A., Kingstone, A., \& Enns, J. T. (in press). In sight, out of mind: The role of eye movements in the rapid resumption of visual search. Perception \& Psychophysics.

Vorberg, D., Mattler, U., Heinecke, A., Schmidt, T., \& SchWARZBACH, J. (2003). Different time courses for visual perception and action priming. Proceedings of the National Academy of Sciences, 100, 6275-6280.

Watson, D. G., \& Humphreys, G. W. (1997). Visual marking: Prioritizing selection for new objects by top-down attentional inhibition of old objects. Psychological Review, 104, 90-122.

Woodman, G. F., \& LucK, S. J. (2004). Visual search is slowed when visuospatial working memory is occupied. Psychonomic Bulletin \& Review, 11, 269-274.

\section{NOTES}

1. Henceforth, we will use the term preprocessing to refer to some limited amount of perceptual processing of one or more items in the search array following one of the presentations of the display. To be clear, we use this term here atheoretically, that is, with no prior commitment to any particular form of neural activity that may be involved.

2 . We also conducted parallel pilot tests in which the orientation of the T was changed randomly between looks and was either task irrelevant (as in all previous experiments) or task relevant. This led to the same conclusions, and so we report only the color change experiments here.

3. In successful masking, a briefly presented target item may initiate an accurate perceptual hypothesis, but this hypothesis cannot be confirmed before the display is replaced by the masking item. The ensuing mismatch between the hypothesis (the target) and the new sensory information (the mask) resets the system and a new hypothesis is initiated based on this new item, which is eventually seen because its hypothesis can be confirmed without further interference.

(Manuscript received May 23, 2006; revision accepted for publication February 5, 2007.) 